\title{
Dynamique des populations larvaires et régime alimentaire de Rhyacophila tristis Pictet (Trichoptera : Rhyacophilidae) dans un ruisseau de haute montagne
}

\author{
P. Lavandier ${ }^{1}$
}

Mots clés : Insecte. Trichoptera. Rhyacophilidae, cycle biologique, nutrition, mortalité, croissance, production.

Rhyacophila tristis Pictet a été étudiée durant 3 années consécutives dans un ruisseau pyrénéen (altitude 1930 m température maximum $17^{\circ} \mathrm{C}$ en août - couvert de neige 4-6 mois par an).

Les larves sont essentiellement carnivores et se nourrissent surtout de chironomidae. Le développement larvaire dure 2 ans. La croissance s'effectue surtout en période déneigée. Entre le ler et le $2 *$ hiver de vie larvaire, l'accroissement (en poids sec) fut en moyenne de $2.25 \% \mathrm{j}^{-1}$. Durant cette période. $1.6 \%$ des larves disparaissaient en moyenne chaque jour. Les taux instantanés de croissance et de mortalité demeurent voisins d'une génération à la suivante. La production a été estimée à $240 \mathrm{mg} \mathrm{m}^{-2}$ an -1 . Le rapport Production/Biomasse moyenne annuelle $=3,1$.

Larval population dynamics and feeding of $R$ hyacophila tristis Plctet Trichoptera : R hyacophilidae) in a high mountain river.

Keywords : Insect, Trichoptera, Rhyacophilidae, life cycle, nutrition, mortality, growth, production.

Rhyacophila tristis Pictet has been studied for three consecutive years in a Pyrenean river (altitude $1930 \mathrm{~m}$. maximum temperature $17^{\circ} \mathrm{C}$ in August, snow cover 4-6 months per year).

The larvae are essentially carnivores. feeding chiefly on chironomids. Larval development takes two years. Growth occurs chiefly in the period when there is no snow. Between the first and second winter of larval life, the growth rate (in terms of dry weight) has a mean value of $2.25 \%$ day $^{-1}$. During this period, the population loss-rate has a mean value of $1.6 \%$ day ${ }^{-1}$. Instantaneous rates of growth and mortality remain similar from one generation to the next. Production has been estimated at $240 \mathrm{mg} \mathrm{m}^{-2}$ year $^{-1}$ (meen annual production to biomass ratio $=3.1$ ).

Rhyacophila tristis Pictet est un trichoptère Rhyacophilidae de petite taille dont les larves appartiennent au groupe hyporhyacophila (Döhler 1950). En Europe l'espèce est largement répandue parmi le rhithron (Botosaneanu et Malicky 1978).

Dans les Pyrénées, elle est présente dans les sources, ruisseaux et rivières entre 600 et $2400 \mathrm{~m}$ d'altitude et forme en haute montagne des populations importantes dans les ruisseaux déversoirs de lac: $c$ 'est une espèce relativement indifférente au régime thermique, qui se rencontre dans des milieux dont la température maximum varie de 8 à $21^{\circ} \mathrm{C}$

1. Laboratoire d'Hydrobiologie, UA 695 du CNRS, Université Paul Sabatier, 118 route de Narbonne, 31062 Toulouse Cedex, FRANCE.
(Décamps 1968). Si les facteurs de répartition de Rhyacophila tristis sont assez bien connus (Moretti et Mearelli 1981), les données biologiques sont à notre connaissance rares. Dans ce travail, nous étudions le régime nutritif et la dynamique du peuplement de l'espèce en haute montagne, à la limite supérieure de sa répartition géographique.

Milieu - Matériel - Méthode

Le ruisseau prospecté correspond à l'affluent $\mathbf{a}_{5}$ du torrent d'Estaragne dont le réseau hydrog raphique a fait l'objet d'une description antérieure (Lavandier 1974).

- La station est située vers $1930 \mathrm{~m}$ d'altitude.

- Le lit, étroit $(0.5 \cdot 1 \mathrm{~m})$, est formé d'une succession de seuils et de mouilles; la vitesse du courant $(10.30 \mathrm{~cm} / \mathrm{s}$ à l'étiage) peut atteindre $200 \mathrm{~cm} / \mathrm{s}$ lors des crues. 
- La température est de $0^{\circ} \mathrm{C}$ sous la neige en hiver : elle s'elève dés le déncigement (juin) et peut atteindre $17^{\circ} \mathrm{C}$ en août avec des amplitudes quotidiennes de 7 à $8^{\circ} \mathrm{C}$. La température diminue ensuite progressivement jusqu'al recouvrement du rujsseau par la neige en décembre.

Trois relevés quantitatifs (filet de Surber - surface prélevée $=0,1 \mathrm{~m}^{2}$ - vide de maille $0,15 \mathrm{~mm}$ ) ont été effectués 2 fois par mois en 1971, I fois par mois en 1972 et 1973. durant la période d'accessibilité du cours d'eau. Compte tenu des résultats obtenus en 1971, 4 séries de 10 relevés ont été effectuées en juillet et novembre 1972 et 1973 afin de mieux cerner l'évolution numérique des populations.

La largeur des capsules céphaliques serı de critère de taille. Les mesures - à $0,05 \mathrm{~mm}$ près - font apparaitre l'existence de 5 stades larvaires (fig. 1). Le poids sec moyen (après séchage à l'étuve à $60^{\circ} \mathrm{C}$ pendant $24 \mathrm{~h}$ ) des 3 premiers stades a été établi par pesée de lots de larves. Les stades IV et $\mathrm{V}$ ont été pesés individuellement.

Nous avons estimé à chaque date d'échantillonnage le nombre moyen $/ \mathrm{m}^{2}$ d'individus des différents stades, la biomasse moyenne $/ \mathrm{m}^{2}$ et le poids individuel moyen des larves (par cohorte lorsque plusieurs coexistent dans le milieu). L'étude de la dynamique du peuplement est basée sur ces éléments.

Le régime alimentaire est déduit des contenus intestinaux d'une centaine de larves capturées en juillet, septembre, octobre et novembre. L'importance du contenu intestinal a été estimé par un indice de réplétion allant de 0 (intestin vide) à 5 (intestin plein).

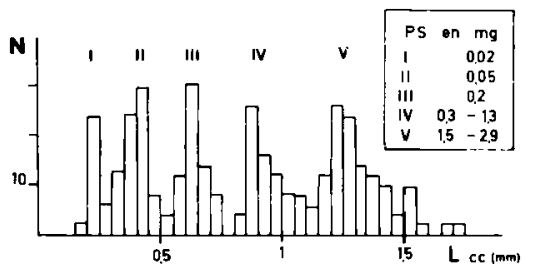

Fig. 1. Longueur des capsules céphaliques ( $L$ c.c) et poids sec des cinq stades larvaires de Rhyacophila tristis.

\section{Régime alimentaire}

L'analyse des contenus stomacaux (Tableau I) conduit aux observations suivantes :

- Le coefficient de réplétion des estomacs est plus fort au milieu qu'au début ou à la fin de la phase déneigée.

- Un estomac sur cinq en moyenne renfermait des débris végétaux en absence de chitine. Cette proportion est plus forte chez les individus jeunes que chez les individus àgés.
Tableau I. Régime alimentaire de Rhyacophila tristis.

\begin{tabular}{|c|c|c|c|c|c|c|c|c|c|c|c|}
\hline \multirow[b]{2}{*}{ Stade larraite } & \multicolumn{3}{|c|}{ Juillet } & \multicolumn{3}{|c|}{ Septembre } & \multicolumn{3}{|c|}{ Novembre } & & \multirow[t]{2}{*}{ Sotal } \\
\hline & II & II! & $\checkmark$ & [ I 1 & IV & $v$ & II & [I] & tv & $v$ & \\
\hline Effectif observes & 4 & 15 & 13 & 3 & 8 & 23 & 2 & 20 & 8 & 9 & 105 \\
\hline $\begin{array}{l}\text { Degre moyen de } \\
\text { repletion }\end{array}$ & 1.1 & 1 & 1,8 & 3 & 4 & 3,8 & 2 & 2,1 & 2 & 2 & \\
\hline $\begin{array}{l}\text { Effectif des proies } \\
\text { ingerees : }\end{array}$ & & & & & & & & & & & \\
\hline 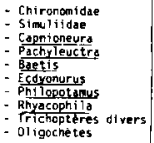 & 1 & $\begin{array}{l}5 \\
1 \\
2 \\
1\end{array}$ & $\frac{1}{2}$ & 3 & $\begin{array}{l}8 \\
\vdots \\
1\end{array}$ & $\begin{array}{l}7 \\
2\end{array}$ & 1 & $\begin{array}{r}18 \\
4 \\
2\end{array}$ & 1 & & $\begin{array}{r}86 \\
6 \\
3 \\
6 \\
13 \\
2 \\
1 \\
1 \\
3 \\
12\end{array}$ \\
\hline
\end{tabular}

- Les proies animales sont déchiquetées et, à l'exception des chironomidae parfois intégralement ingérés, difficilement identifiables. Dix taxa ont néanmoins été reconnus (Tableau I) : ce sont les principales populations du milieu (Lavandier 1979), hormis les formes carnivores (Perla, Isoperla, Plectrocnemia) et les Trichoptères à fourreaux (Synagape(us, Thremma, Micrasema); les chironomidae constituent toujours la plus grande partie des captures même quand ce sont les Baetidae qui dominent parmi le benthos.

- Les soies d'oligochètes sont fréquentes dans le tractus digestif des jeunes mais ne sont pas observées chez des individus âgés.

- La taille des chironomidae ne semble pas liée a celle du predateur. L'estimation de la taille des autres taxa est le plus souvent irréalisable car les griffes ou les pieces buccales susceptibles de servir de base à une relation biomét rique paraissent rarement consommées ; toutefois des fragments de Bae. tis âgées identifiés dans l'intestin de deux larves au stade III, montrent que la dimension des invertébrés ingérés peut ètre importante par rapport à celle du prédateur.

\section{Développement larvaire}

Le développement larvaire est déduit de l'évolution de la structure des populations au cours de l'année (fig. 2).

Les éclosions ont lieu en automne; les premières larvules peuvent at teindre le stade III à la fin de l'année. Le développement est ralenti en hiver, faible au printemps, plus rapide en été. Les premières nymphes sont recueillies au début du deuxième hiver passé aux stades IV, V et nymphal. Lors de la 


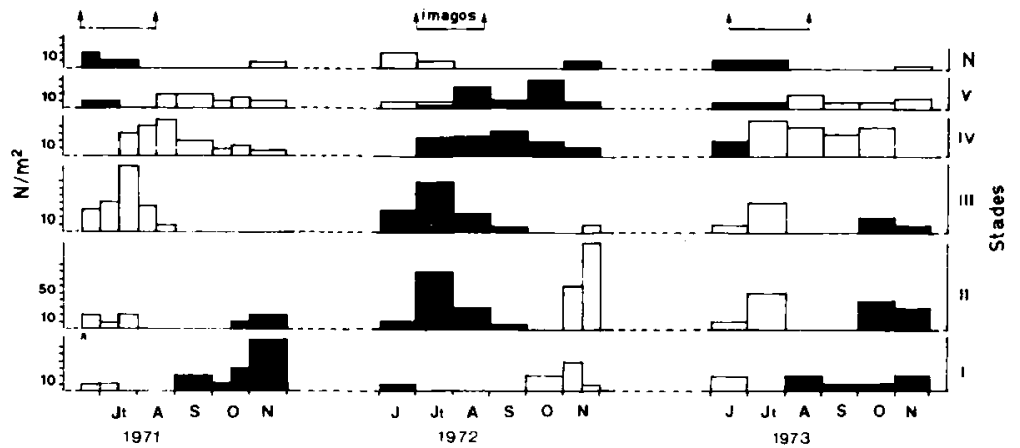

Fig. 2. Cycle biologique de Rhyacophila tristis. Periodes de vol et Nombre d'individus/m² des cinq stades larvaires (I. à $\mathrm{V}$ ) et nymphal $(\mathrm{N})$ aux différentes dates d'échantillonnage.

crue nivale, seuls subsistent les deux derniers stades de la génération. Les adultes s'envolent en juillet.

Ce schéma de développement se décale selon les conditions climatiques annuelles : en 1972 la croissance fut plus tardive que les autres années (quatre stades larvaires subsistaient encore en septembre et la nouvelle génération n'apparut qu'en octobre). Ce retard a toutefois été compensé par la précocité du déneigement au printemps suivant et n'a eu aucune répercussion sur la période de vol de $R /$. tristis.

\section{Evolution numérique}

Plusieurs phases se succèdent entre l'éclosion et l'émergence: (1) les effectifs s'accroissent en fin d'année avec l'éclosion des cufs. L'effectif maximum s'observe après l'hiver, à la fin de la fonte des neiges. (2) Les effectifs s'abaissent de façon exponentielle en fonction du temps au cours de la période déneigée ( $f$ ig. 3) ; la corrélation entre le logarithme des effectifs moyens $/ \mathrm{m}^{2}$ et le temps est significative au seuil $\mathbf{P}=0,1$ pour les générations nées en 1970 et 1972 , et au seuil $P=0,01$ pour la génération née en 1971). Les taux de diminution des nombres $(\%$ effectif $j^{-1} \pm 95 \%$ L.C.) pour les 3 générations successives furent respectivement de $1,55 \pm 1,7 \% \mathrm{j}^{-1}$ $(n=8), 1,66 \pm 0,6 \% j^{-1}(n=6)$ et $1,2 \pm 1,6 \% j^{-1}$ $(n=5)$; ils ne présentent pas de différences signifi- catives. (3) Les effectifs se maintiennent au cours du deuxième hiver, puis se raréfient avec l'envol des imagos.

Les estimations numériques plus précises faites en juillet et novembre 1972 et 1973 montrent qu'en fin d'année, les effectifs néonates peuvent fluctuer assez largement d'une année sur l'autre (les nombres de larvules sont significativement différents en novembre 1972 et novembre 1973). Après le premier hiver en revanche les générations nées en 1971 et en 1972 ne présentaient pas de différences d'effectifs significatives pour des périodes homologues de la vie larvaire.

\section{Croissance pondérale}

Quatre phases de croissance peuvent être distin. guées au cours de la vie larvaire ( $F \mathrm{ig.} \mathrm{3)} \mathrm{:} \mathrm{(1)} \mathrm{En} \mathrm{début}$ de cycle biologique, l'évolution du poids individuel moyen des larves néonates est irrégulière. (2) La croissance est très faible au cours de la période hivernale. (3) Le développement reprend dès le dénejgement et, jusqu'à l'hiver suivant, il existe une rela. tion hautement significative entre le logarithme du poids individuel moyen des larves à chaque date de prélèvement et le temps. Durant cette période les taux de croissance ( $\%$ poids sec $\pm 95 \%$ L.C.) furent respectivement de $1,93 \pm 0,44 \% \mathrm{j}^{-1}(\mathrm{n}=10), 2,4$ $\pm 0,42 \% j^{-1}(n=7)$ et $2,45 \pm 0,35 \% j^{-1}(n=6)$ pour les générations nées en 1970, 1971 et 1972. Ces 

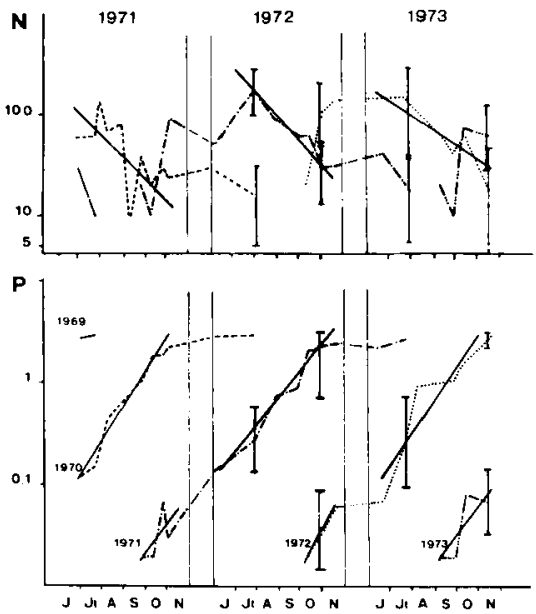

Fig. 3. Evolution du nombre d'individus $\left(\mathrm{N} / \mathrm{m}^{2}\right)$ et du poids individuel moyen $(P=$ poids sec en $\mathrm{mg}$ ) des laries de chaque cuhorte au cours des Irois années d'études (L.C. $95 \%$ sont indiquées pour les estimations de juillet el novembre 1972 et 1973).

valeurs ne présentent pas de différences significatives. (4) La croissance pondérale est ensuite faible jusqu'à la fin du cycle biologique du fait des nouvelles conditions hivernales, de la nymphose et de l'envol des imagos.

Si on applique le mème modèle de croissance à toutes les phases du développement, le taux de croissance des larves néonates $\left(2,25 \% \mathrm{j}^{-1}\right.$ de valeur moyenne) est analogue à celui des larves plus âgés. En période hivernale et quel que soit l'âge des larves, les taux de croissance sont beaucoup plus faibles : $0,16 \% \mathrm{j}^{-1}$ en moyenne.

\section{Production - Biomasse}

La production est calculée par la méthode graphique d'Allen (1951) à partir d'une courbe unique de croissance-survie, basée sur les caractéristiques des différentes cohortes qui se sont succédées au cours des trois années d'ètude (cf. Lavandier 1982). La production moyenne annuelle a été évaluée à $240 \mathrm{mg}$ $m^{-2}$. La biomasse moyenne en période déneigée était de $70 \mathrm{mg} \mathrm{m}^{-2}$. Compte tenu de la structure des populations en hiver, la biomasse moyenne annuelle a pu être estimée à $77 \mathrm{mg} \mathrm{m}^{-2}$ et la biomasse moyenne d'une cohorte au cours de son développement à $41 \mathrm{mg} \mathrm{m}^{-2}$.

\section{Discussion}

Rhyacophila tristis Pictet est une espèce essentiellement carnivore. Le matériel végétal et les détritus, surtout présents chez les stades jeunes, ne constituent toujours qu'une faible part du contenu digestif. Ce régime est conforme à celui de la grande majorité des espèces de Rhyacophila. Les travaux de Décamps 1967 b, Cummins 1973, Wiggins 1977, Martin et Mackay 1982, recensent la plupart des observations faites sur ce sujet. Rhyacophila tristis ingère préférentiellement des chironomidae et il a été montré expérimentalement que la sélection des proies pouvait influer sur la croissance larvaire (Martin et Mackay 1983) et déterminer en partie la microdistribution des espèces.

La détermination des modalités de développement à partir de la structure des populations larvaires est souvent difficile chez les Rhyacophila car plusieurs stades larvaires coexistent fréquemment. Les espèces étudiées en Amérique paraissent univoltines (Thut 1969, Mackay 1969, Karl et Hilsenhoff 1979, Manuel et Folson 1982), mais plusieurs cohortes peuvent se succéder au cours de l'année. Les travaux relatifs aux espèces européennes signalent des durées de développement variant de 1 à 3 ans selon les espèces et les condit ions de milieu -1 an : Rhyacophila denticula Mc L. (Lavandier et Dumas 1971), $R$. mocsaryi Klap, $R$. occidentalis Mc L. (Décamps 1967), $R$. obliterata Mc L. (Hynes 1961, Crichton et Fisher 1978) ; 1 ou 2 ans : $R$. dorsalis Curtis (Scott 1958, Mackereth 1960, Hynes 1961, Elliott 1968), $R$. munda Mc L. (Elliott 1968); 2 ans : $R$. nubila zett. (Ulfstrand 1968), $R$. angelieri Décamps (Décamps 1967. Lavandier 1979) ; 1 à 3 ans : $R$. evoluta Mc L., $R$. intermedia Mc L. (Décamps 1967, Lavandier 1979). Dans les Pyrénées, Décamps (1967) a souligné la variabilité du développement des espèces de montagne et leur capacité à passer la mauvaise saison à n'importe quel stade de leur cycle. C'est ici le cas de Rhyacophila tristis Pjctet, dont tous les stades larvaires doivent subir une période hivernale au cours du cycle biologique. 
Malgré la répartition contagieuse des larves et leurs faibles effectifs, nos relevés donnent une bonne image de la dynamique des populations. Les estimations réalisées en début et en fin de la période déneigée à partir d'échantillonnages fournis encadrent parfaitement les résultats mensuels basés sur une moyenne de 3 relevés seulement.

L'effectif initial des diverses cohortes et par suite la mortalité en début de cycle biologique, n'a pu être estimé compte tenu de l'étalement et de la position dans le temps de la période d'éclosion. Les rares données relatives aux trichoptères (Elliott 1981-1982) indiquent une mortalité importante en début de développement. Une fois les éclosions achevées et après la fonte des neiges au cours de laquelle les larves sont difficiles à capturer (Lavandier et Décamps 1983), les effectifs sont chaque année du mème ordre et sur 100 larves présentes en juillet (stades I, II et III) 15 à 20 subsistent en fin d'année (stade IV, $V$ et VI). La mortalité est ensuite faible jusqu'à l'émergence.

La croissance, qui est très faible en hiver et qui s'effectue essentiellement durant la période déneigée, paraît en grande partie liée à la température. Toutefois, même en été, les taux de croissance instantanés, qui sont au maximum de $2,5 \% \mathrm{j}^{-1}$, demeurent relativement faibles et sont parmi les plus bas calculés jusqu'à présent chez les trichoptères (Anderson 1977, Lavandier 1979, 1984, Elliott 1981, 1982). L'étude des populations de $R$. tristis vivant à plus basse altitude, permettrait de mieux comprendre les caractéristiques de la croissance, en précisant en particulier l'influence du régime alimentaire, de la température et vraisemblablement du régime des eaux sur la vitesse du développement.

Les estimations de production faites sur une vingtaine d'espèces de trichoptères varient de 0,009 à $2,8 \mathrm{mg} \mathrm{m}^{-2}$ an $^{-1}$ (cf. synthèse de Waters 1977 . Elliott 1981, 1982, Lavandier 1979, 1984). Parmi les 13 espèces étudiées dans le réseau hydrographique de l'Estaragne, une espèce (Allogamus auricollis, Pictet) présentait une production supérieure à $2,5 \mathrm{mg}$ $\mathrm{m}^{-2}$ an -1 ; la production des 12 autres espèces était comprise entre $0,018 \mathrm{~g} \mathrm{~m}^{-2}$ an ${ }^{-1}$ (Thremma gallicum Mc $\mathbf{L}$.) et $1,25 \mathrm{~g} \mathrm{~m}^{-2}$ an $^{-1}$ (Drusus rectus Mc L.). La production de Rhyacophila tristis Pictet est égale à cette valeur moyenne : elle constitue $3 \%$ de la production benthique dans le ruisseau étudié (Lavandier et Décamps 1983).
Le rapport production annuelle/biomasse moyenne en période déneigée $(=3,4)$ est voisin des rapports établis en Estaragne sur des populations dont le développement s'étend sur deux années. Le rapport production/biomasse moyenne d'une cohorte $(=5,8)$ est légèrement supérieur à la valeur 5 proposée par Waters (1979) pour les calculs de production ; de même, le rapport production/biomasse moyenne annuelle $(=3,1)$ est plus ćlevé que la valeur moyenne $(2,2)$ observée pour les espèces hémivoltines du zoobenthos (Waters 1977).

\section{Travaux cités}

Allen (K.A.). 1951, - The Horokiwi stream. A study of a truut population. Fish. Bull. N.Z., $10: 1-231$.

Anderson (N.H.). 1977. - Continuous rearing of the limnephilid caddislly. Clistoronia magnifica (Banks). Proceedings of the $2^{\text {th }}$ International Symposium on Trichoptera, 317-329.

Botosaneranu (L.) \& Malicky (H.). 1978. - Trichoptera. In ; Limnofauna Europaea, Illies J. (ed.) G. Fischer Verlag. Stuttgart 333-359.

Crichton (M.I.) \& Fisher (D.). 1978, - Life histories and distribu. tion of British Trichoptera, excluding Limnephilidae and Hydroptilidae, based on the Rothamsted Insect Survey. Holarct. Ecol., 1 : 31.45.

Cummins (K.W.). 1973. - Trophic relations of aquat ic insects. Ann. Rev. Entomol., $15: 25-42$.

Décamps (H.). 1967. - Ecologie des Trichoptères de la vallée d'Aure (Hautes-Pyrénées). Annls Limnol., 3 (3) : $399-577$.

Décamps (H.). 1968. - Vicariances écologiques chez les Trichoptères des Pyrénées. Annls. Limnol, 4 (1): 1-50.

Döhler (W.). 1950. - Zur Kenntris der Gattung Rhyacophila im mittel-europäischen Raum (Trichoptera). Arch. Hydrobiol., 44 : 271-293.

Elliott (J.M.). 1968. - The life histories and drifting of Trichoptera in a Dartmour stream. J. Anim. Ecol, $37: 615-625$.

Elliott (J.M.) 1981. - A quantitative study of the life cycle of the net-spinning caddis Philopotamus monianus (Trichoptera: Phjlopotamidae) in a lake district stream. I. Anim. Ecol., 50: $867-883$.

Elliott (J.M.). 1982. - A quantitative study of the life cycle of the case building caddis Odontocerum albicome (Trichoptera : Odontoceridael in a lake district stream. Freshwat. Biol., 12 : 241-255.

Hynes (H.B.N.). 1961. - The invertebrate fauna of a wel sh mountain stream, Arch. Hydrobiol., $57: 344-388$.

Karl (T.S.) \& Hilsenhoff (W.L.), 1979. - The caddisflies (Trichoptral of Parfiey's Glen Creek, Wisconsin. Trans. Wisc. Acad. Sci. Ants Leti, $67: 31-42$.

Lavandier (P.). 1974. - Ecologie d'un torrent pyrénken de haute montagne. I. Caractéristiques physiques. Annls Limnol., 10 (2) 173.219.

Lavandier (P.). 1979. - Ecologie d'un torrent pyrénéen de haute montagne : I'Estaragne. Thèse sciences Univer'sité Paul Sabatier Toulouse : $532 \mathrm{p}$.

Lavandier (P.). 1982. Développement larvaire. Régime alimentaire Production d'/soperla viridinervis Pictet (Plecoptera, Perlodidae) dans un torrent froid de haute montagne. Annis Limnol. $18(3): 301 \cdot 318$. 
Lavandier (P.). 1984. - Dynamique des populations larvaires d'Apatania siylata (Trichoptera) dans un ruisseau pyrénéen de haute montagne. Bull. Soc. Hist. Nat. Toulouse, 120:95-98.

Lavandier (P.) \& Dumas (J.). 1971. - Cycles de développement de quelques invertébrés benthiques dans des ruisseaux des Pyré. nées centrales. Annls Limnol. 7 (2): 157.172.

Lavandier (P.) \& Decamps (H.). 1983. - Un torrent d'altitude dans les Pyrénées: l'Estaragne. In : Problèmes d'écologie : Ecosys. tèmes Limniques. Lamotte et Bourlière (eds), Masson, Paris : 81-111.

Mackay (R.J.). 1969. - Aquatic insect communities of a small stream on Mont St Hilaire, Québec. J. Fish. Res, Board Can., $26(5): 1157.1183$

Mackereth (J.C.). 1960. - Notes on the Trichoptera of a stony stream. Proc. R. ent. Soc. Lond, A, 35, 17.23.

Manuel (K.L.) \& Folsom (T.C.). 1982. - Instar sizes, life cycles and food habits of five Rhvacophila (Trichoptera : Rhyacophilidae) species from the Appalachian Mountains of South Carolina, U.S.A. Hydrobiologia, 97 (3): $281-285$.

Martin (I.D.) \& Mackay (R.J.). 1982. - Interpreting the diet of Rhyacophila larvae (Trichoptera) from gut analyses : An evaluation of techniques. Can. J. Zool., $60: 783-789$.
Mar1in (1.D.) \& Mackay (R.J.). 1983. - Growth rates and prey selection of two congeneric predatory caddisflies (Trichoptera: Rhyacophilidae), Can. J. Zool., 61 (4) : 895-900.

Moretti (G.P.) \& Mearelli (M.). 1981. - Ecological profiles in three Rhyacophila species. In Third international Symposium on Trichoptera. Moretti G.P. (ed.). W. Junk the Hague 227-230.

Scott (D.). 1958. - Ecological studies on the Trichoptera of the River Dean. Cheshire, Arch. Hydrobiol., $54: 340-392$.

Thut (R.N.) 1969. - Feedings habits of larvae of seven Rhyacophile (Trichoptera : Rhyacophilidae) species with notes on other lifehistory features. Ann. Am. Entomol. Soc. 62 : 894-98.

Ulfstrand (S.). 1968. - Life cycles of benthic insects in Lapland streams (Ephemeroptera, Plecoptera. Trichoptera, Diptera Simuliidaek : Oikos $19: 167-190$.

Waters (T.F.). 1977. - Secondary production in inland waters. In : Advances in ecological research Macfayden A. (ed.). Vol. 10: 91-164. Academic Press London.

Waters (T.F.). 1979. - Influence of benthos life history upon the estimation of secondary production. J. Fish. Res, Board Can., $36(12) \div 1425 \cdot 1430$.

Wiggins (G.B.). 1977. - Larvoe of the North American Caddisfly genera (Trichoptera). University of Toronto Press TorontoBuffalo: $401 \mathrm{p}$. 